\title{
Variations
}

Variations

Revue internationale de théorie critique

$24 \mid 2021$

Echos

\section{L'art antistalinien}

Benjamin, Chostakovitch et la fin de l'histoire

\section{Susan Buck-Morss}

Traducteur : David Buxton et Christophe Magis

\section{OpenEdition}

Journals

Édition électronique

URL : https://journals.openedition.org/variations/1900

DOI : 10.4000/variations. 1900

ISSN : 1968-3960

Éditeur

Les amis de Variations

Référence électronique

Susan Buck-Morss, «L'art antistalinien », Variations [En ligne], 24 | 2021, mis en ligne le 03 juillet 2021, consulté le 07 juillet 2021. URL : http://journals.openedition.org/variations/1900 ; DOI : https:// doi.org/10.4000/variations. 1900

Ce document a été généré automatiquement le 7 juillet 2021.

Les ami•e•s de Variations 


\title{
L'art antistalinien
}

\author{
Benjamin, Chostakovitch et la fin de l'histoire
}

\author{
Susan Buck-Morss
}

Traduction : David Buxton et Christophe Magis

\section{NOTE DE L'ÉDITEUR}

Ce texte est la transcription du discours-programme du premier congrès de l'Association internationale Walter Benjamin qui a eu lieu à Amsterdam en juillet 1997. Publiée sous le titre « Revolutionary Time : The Vanguard and the Avant-Garde » dans Benjamin Studies, Studien 1, (dir. Helga Geyer-Ryan), Amsterdam, Rodolphi, 2002.

Note des traducteurs : La traduction essaie de respecter la dimension orale du texte. La langue française ne permet pas de distinguer entre l'avant-garde artistique (avantgarde) et politique (vanguard). Ce dernier terme a été systématiquement traduit par « avant-garde politique »; en l'absence de précisions, « avant-garde » réfère à l'avantgarde artistique.

1 Je ne suis pas la première à pointer l'ironie de notre présence ici, intellectuels rassemblés à l'occasion d'un congrès international sur Walter Benjamin! Mais pour paraphraser le Grand Penseur lui-même, si l'on peut accepter qu'il existe des congrès benjaminiens, il y a quand même une différence importante entre un bon congrès benjaminien et un mauvais - je paraphrase ici ses propos à propos de l'adaptation cinématographique de Faust. Celui-ci a été particulièrement réussi, grâce à l'énergie positive déployée par les directeurs de l'Association internationale Walter Benjamin. En tant que dernière personne à intervenir dans les séances plénières, j'aimerais les remercier de notre part à toutes et à tous.

2 Pourtant, une question se pose. Le présent congrès international consacré à Walter Benjamin, organisé sous l'égide d'une Académie qui l'avait rejeté et honorant un Grand Penseur qui avait implacablement dénigré l'idée même d'un culte du génie n'est-il pas par ailleurs facilité par la puissance mondiale d'un capitalisme moins tardif que subsistant à perpétuité - cette même force que Benjamin accusait de réprimer le 
potentiel humain de la technologie. Tout cela n'est-il pas excessivement contradictoire ? Et, si oui, alors peut-on essayer de dépasser ces contradictions vers une compréhension dialectique de ce que nous faisons ici? Et cela évidemment, en supposant que nous savons tous ce que veut dire "dialectique». Pour ma part, cependant, après avoir écrit deux livres avec ce mot dans le titre, je n'en suis plus certaine...

Un aspect - appelons-le dialectique - de la théorie de l'École de Francfort en général, et de Walter Benjamin en particulier, qui marque le siècle et qui continue de nous fasciner, maintenant plus que jamais, réside dans l'articulation d'une politique radicale, sociale-révolutionnaire, et d'une méfiance absolue de l'Histoire comme progrès réalisant ainsi la synthèse de deux positions pensées jusqu'alors comme s'opposant. Traditionnellement, c'était la gauche socialiste qui croyait en le progrès historique, tandis que la droite conservatrice se présentait comme un ensemble de critiques nostalgiques du cours de l'Histoire. Mais dans ce $\left[20^{e}\right]$ siècle, la politique révolutionnaire et le pessimisme historique ont fini par se rencontrer, l'intégrité intellectuelle n'ayant pas permis autrement. Il était impossible d'être passé à travers ce siècle qui touche de façon chaotique à sa fin et de maintenir une croyance intacte, ni en le capitalisme comme réponse aux prières des pauvres, ni en l'Histoire comme réalisation de la Raison. Les contre-exemples sont trop nombreux sur tous les continents. Au sein de chaque groupe ethnique, et de chaque civilisation mondiale, les atrocités commises ont été, et continuent d'être barbares, qu'elles soient effectuées à coups de hache et de machette ou par des moyens technologiques de plus en plus sophistiqués. Entre-temps, comme grise toile de fond de ces évènements politiques, le fossé économique entre riches et pauvres non seulement persiste, mais devient abyssal, situation que la nouvelle organisation mondiale du capitalisme - vainqueur indiscuté de l'Histoire - ne cherche même plus à excuser. Si le " progrès » historique nous donne le capitalisme, et si le capitalisme ne peut nous donner une organisation raisonnable de la société, alors on ne peut qu'être inexorablement conduit vers la position benjaminienne ou francfortienne.

4 Inexorablement. Je rejette à dessein ici le pluralisme politique. Comme une de mes enseignantes - qui était d'ailleurs, comme par hasard, émigrée socialiste de l'Allemagne nazie - me l'a un jour expliqué, « les libéraux sont si ouverts que leurs cerveaux tombent de leurs crânes ». Alors, j'insiste: l'intégrité intellectuelle exige notre engagement à la fois dans une critique radicale du capitalisme et dans une critique radicale du progrès historique. Cela peut se faire à partir d'une pluralité de positions sociales - constructions de race, de sexualité, d'ethnicité, de postcolonialité, etc. -, mais cela ne peut en aucun cas se faire de manière confortable. Trop de confort et nous faisons nous-mêmes partie du problème, soit en tant que mandarins, spécialistes reconnus de Benjamin parcourant le monde, soit en tant que jeunes chercheurs, groupies des premiers. Je parle ici de l'inconfort intellectuel plus que de l'inconfort financier, bien que les deux soient souvent liés. Je m'adresse en particulier à la jeune génération de Benjaminiens dans le public qui se trouve en état d'inconfort permanent, attirée (espérons-le) par les écrits de Benjamin en raison de leur radicalité intellectuelle, mais condamnée à se bousculer pour les quelques postes réservés plutôt aux candidats les plus opportunistes ou les plus circonspects. Ceci est notamment vrai aux États-Unis, où le système universitaire, sous l'influence des institutions privées, a adopté les "bonnes» pratiques du monde de l'entreprise : réduction du personnel enseignant et accroissement de leur charge de travail, fermeture des départements 
« inefficaces », non rentables, remplacement d'une partie du personnel administratif par des programmes informatiques, augmentation des frais d'inscription pour les étudiants-consommateurs et, plus radicalement, menace de supprimer les postes titulaires, afin de remplacer les enseignants libres par des doctorants vulnérables à un coût sensiblement plus faible. Si cette logique continue d'être incontestée, la situation deviendra insupportable. Les compromis réclamés par un système universitaire en voie de rétrécissement à une vie intellectuelle qui aspire à être libre deviendront trop grands. Quelque chose va casser! On ne sait pas qui profitera de cette situation. Cela dépendra de ce que nous, intellectuels, faisons collectivement - en tant que classe, pourrait-on dire. Autrefois, on disait « socialiste » pour désigner une action collective de classe ; c'est un mot qu'il faut réhabiliter ! Contre ceux qui rejettent la politique socialiste comme une relique du passé récent, je me permets un argument épistémologique, dialectique, et même à vrai dire hégélien : des formes de socialisme continueront d'être réinventées, car la logique du capitalisme l'exige. La logique sociale déformée du capitalisme rend inévitable la proposition d'alternatives socialistes, car la Raison humaine ne peut être satisfaite sans celles-ci.

Le défi pour les titulaires parmi nous réside dans cette demande dialectique que nous devons nous imposer à nous-mêmes: nous devons transmettre à la prochaine génération une tradition de pensée radicale. Cette demande est dialectique à cause de la contradiction apparente qu'elle fait naître : en quoi la transmission d'une tradition peut-elle être considérée comme un acte radical? La réponse à cette question ne demande rien moins qu'une philosophie de l'Histoire. Et ceux d'entre nous à l'université qui lisent les textes du passé, quelle que soit leur affiliation disciplinaire, sont des historiens, des anges de l'Histoire, du moins dans le sens positionnel : tournés vers le passé, nous avançons vers l'avenir.

6 Ce qui rend la philosophie de l'Histoire de Benjamin si utile pour cette tâche, c'est qu'elle refuse l'opposition binaire entre historicisme et universalisme. Le sens dans l'Histoire n'est ni le «ce qui s'est réellement passé » de von Ranke ("wie es eigntlich gewesen ist"), ni la vérité éternelle, transcendantale, accessible à toutes les époques ${ }^{1}$. Le sens historique est transitoire : il dépend moins du passé que du présent et de l'état du monde réel. Ainsi, on ne peut l'approcher par un simple exercice académique, comme s'il concernait une espèce d'humanoïdes vivant jadis sur Mars. Nous sommes dans l'Histoire, et le temps de cette dernière n'est pas achevé. Nous faisons de l'Histoire dans les deux sens temporels, le passé et le présent. Ce que nous faisons ou ne faisons pas crée le présent ; ce que nous savons ou ne savons pas construit le passé. Ces deux tâches sont inextricablement liées : la façon dont on construit le passé détermine comment on comprend le cours du présent. Pour reprendre la métaphore de Benjamin, le vent de l'Histoire mondiale souffle depuis le passé. Les mots sont comme des voiles de bateau ; leur transformation en concepts dépend de la dynamique de leur déploiement. La causalité de l'Histoire relève de l'action différée (nachträglich) plutôt que des étapes séquentielles au long d'un continuum temporel. Nous produisons cette causalité dans le présent à travers le sens donné aux évènements passés, situation qui entraîne une responsabilité énorme. Ce que nous voyons dans le passé, et comment nous le décrivons, tout cela est d'une importance profonde. Puisque les constructions potentielles de l'Histoire sont pratiquement infinies - et puisque la mer du présent n'a pas de rivages -, il est impossible de savoir à l'avance quelle sera la meilleure manière de procéder à son étude. Peut-être notre responsabilité est-elle en effet de toujours 
rechercher une autre voie, contestant non les faits mais la manière dont ils sont reliés, modifiant en permanence les constellations qui les font apparaître.

7 Constellations. Voilà une autre métaphore de Benjamin, qui relie ses premiers écrits métaphysiques à ses travaux matérialistes plus tardifs. Elle est au centre de sa théorie de la vérité et, pour moi, cette métaphore a été très suggestive. Si nous comprenons les étoiles comme des données empiriques (des faits et des fragments du passé), pratiquement illimitées et intemporelles, alors notre tâche scientifique en tant qu'universitaires est de les découvrir (je crois toujours en le travail d'archive), tandis que notre tâche philosophique et donc politique (comme Benjamin, je mets ces deux mots sur un pied d'égalité) est de relier ces fragments et ces faits dans des figures qui soient lisibles dans le présent, produisant des "constellations » comme autant de variantes de la vérité (et c'est le travail d'archive qui autorise encore l'emploi de ce mot). Dans une société idéale, dit Benjamin, toutes les étoiles seraient incluses, et toutes les constellations seraient déchiffrables. Mais dans la nôtre, ce n'est pas le cas. Le pouvoir déforme notre vision des cieux, en concentrant ses grands télescopes sur des régions particulières, magnifiant leur importance et occultant les autres au point qu'elles ne soient plus même visibles. Un tel pouvoir n'est pas imposé seulement par l'État. Il est inscrit dans la structure même de nos disciplines académiques, qui sont elles-mêmes des dispositifs de grossissement favorisant l'insertion des découvertes dans les constellations discursives déjà portées sur la carte, et ne déplaçant leur point de mire que lentement aux cours des évènements. On fait preuve d'esprit critique quand on refuse d'être contraint par les signes astrologiques dominants. Mais nous choisissons d'ignorer les faits (les étoiles), autant que les tendances de l'époque, à nos risques et périls - notamment si l'on veut déployer les voiles contre le courant. Dans une approche benjaminienne, il ne suffit pas de créer d'autres constellations comme l'histoire des femmes, des Noirs, etc. Les faits déterrés par ce genre d'études devraient faire exploser le continuum culturel, et non le remplacer par un autre ${ }^{2}$. Ce ne sont pas les fins, mais plutôt les étoiles qui nous guident, laissant encore indéterminés le déploiement des voiles, et même la direction du voyage.

Dans cet esprit, à savoir que les fragments déterrés du passé s'insèrent dans de nouvelles constellations dans le présent, je voudrais faire la proposition suivante : la vision changée des cieux de l'Histoire après la fin de la Guerre froide pourrait permettre le traçage d'autres lignes de connexion, pertinentes non seulement pour interpréter la biographie intellectuelle de Benjamin, mais aussi la biographie (si on peut parler ainsi) de la gauche révolutionnaire.

Dans les disciplines établies, on nous a appris à comprendre Walter Benjamin dans le contexte des développements historiques en Europe occidentale : au sein du marxisme européen, du surréalisme français, de la culture du Weimar, et de la pensée germanojuive. Mes propres travaux font partie de cette tradition. Mais Benjamin lui-même n'a pas vécu Guerre froide. Pour lui, du moins après sa rencontre avec Asja Lacis en 1924, les questions intellectuelles brûlantes étaient posées par la pratique politique à gauche, sans égard des lieux ethniques et géographiques ${ }^{3}$. Et cette pratique avait lieu le plus intensément (quoique de manière problématique) en Union soviétique. Je ne peux accepter l'argument de Gershom Scholem selon lequel Benjamin aurait «perdu toutes ses illusions » vis-à-vis du socialisme soviétique lors de son voyage à Moscou pendant l'hiver de 1926-274. (Rappelons qu'il a effectivement fait ce voyage, alors que, malgré des promesses répétées à Scholem, il ne s'est jamais rendu à Jérusalem, et malgré le 
titre mélancolique d'une œuvre tardive, Central Park, il n'a pas suivi l'École de Francfort à New York). Les écrits de Benjamin, contra Scholem, font montre de l'importance continue du socialisme soviétique pour sa pensée.

10 Au milieu des années 1930, bien après son séjour à Moscou, le travail de Benjamin témoigne d'une conscience des discussions critiques qui ont eu lieu parmi les artistes soviétiques depuis plus d'une décennie. Cela ne se ressent pas uniquement dans la courte intervention titrée "L'auteur comme producteur ", prononcée à Paris en 1934 à l'Institut pour la recherche sur le fascisme - une organisation communiste ${ }^{5}$ - mais également dans son célèbre essai, cité à tort et à travers, écrit en 1935 et publié en 1936, présenté par Benjamin lui-même comme "la théorie matérialiste de l'art ». L'essai en question, "L'œuvre d'art à l'époque de sa reproductibilité technique », est pourtant encore lu, du moins aux États-Unis, comme une défense dépolitisée de l'industrie culturelle. En fait, dans cet essai, et de manière encore plus explicite dans l'exposé de $1935 \mathrm{du}$ travail sur les passages parisiens, Benjamin décrit comment les nouvelles technologies ont permis la libération des "formes créatives ${ }^{6}$ de l'emprise des arts traditionnels, ce qui résonne indubitablement avec l'affirmation de l'avant-garde bolchévique de la " tendance vers la liquidation de l'art en tant que discipline à part " ${ }^{7}$, dans la mesure où l'art est technologiquement produit. La mise en avant par Benjamin du potentiel cognitif du cinéma comme mode d'enquête épistémologique prend comme exemple le film expérimental de Dziga Vertov, L'Homme à la caméra (1929) ${ }^{8}$. L'essai de Benjamin à propos de l'« Cuvre d'art» adopte un point de vue positif sur ce que l'avant-garde russe appelait dans les années 1920 "l'art-production ", c'est-à-dire l'art qui s'insère dans la vie quotidienne à travers la production industrielle. «L'Auteur comme producteur » emprunte l'idée de "l'artiste-ingénieur ", terme inventé par les constructivistes russes, afin de décrire l'appel de l'artiste au «redéploiement » des appareils techniques de la production culturelle ${ }^{9}$. Quand Benjamin rejette le culte du génie individuel, et annonce le déclin de la division du travail entre producteurs culturels et publics-consommateurs, il fait écho à la position des Proletkult, les organisations culturelles prolétariennes des années 1920 qui, en prônant « l'amateurisme créatif », ont pris position contre l'élitisme culturel du Parti.

11 Benjamin avait beaucoup d'intérêts communs avec l'avant-garde soviétique, de son appréciation de Charles Fourier, largement lu en Russie après la Révolution ${ }^{10}$, à ses théories de la mimesis et de l'innervation qui résonnent de manière fascinante avec les discussions à propos de la biorythmique et de la biomécanique chez des metteurs en scène du théâtre et du cinéma comme Meyerhold et Eisenstein ${ }^{11}$. Même une idée aussi excentrique en apparence que sa théorie anthropomorphique des objets, qui horripilait tant Bertolt Brecht, à savoir que les choses vous regardent quand vous croyez les regardez, est similaire de façon frappante aux spéculations utopiques de l'avant-garde sur «l'objet socialiste » qui devait remplacer la marchandise capitaliste ${ }^{12}$. Rodchenko a écrit à Moscou depuis Paris dans l'été de 1925 (où il visitait l'Exposition internationale des arts décoratifs) ${ }^{13}$, parlant d'une sorte d'aura socialiste dans laquelle «les choses deviennent compréhensives, deviennent des amis et des camarades de la personne, qui apprend à rire et à être heureuse, et à converser avec les choses $»^{14}$.

Bien entendu, aucun des textes de Benjamin, ni l'essai sur l'« Cuvre d'art » ni celui sur l'« Auteur comme producteur », n'approuve le réalisme socialisme tel qu'il était dicté en Union soviétique et disséminé internationalement par le Comintern. C'était déjà le cas quand il s'est rendu à Moscou en 1926: Benjamin n'a jamais assimilé la pratique 
artistique socialiste à la ligne officielle du Parti communiste. Mais les artistes soviétiques non plus. Ni en 1926, ni en 1936 d'ailleurs, bien que la sanction de ce refus ait commencé à devenir effroyablement sévère dans certains cas. Le récent travail d'archive fourni par des historiens russes autant que non russes corrige la compréhension simpliste, issue de la Guerre froide, selon laquelle l'art communiste était soi-disant dicté politiquement d'en haut de façon dogmatique. En effet, les chercheurs comme Franco Borsi ont démontré que les éléments précédemment identifiés comme des marques de "l'art totalitaire» - monumentalisme, néoclassicisme, ordre par répétition - peuvent se trouver généralement dans les œuvres européennes des années 1930, aussi bien dans les sociétés démocratiques que dans les régimes dictatoriaux ${ }^{15}$. Les interrelations complexes entre culture et politique en Union soviétique révélées dans les recherches récentes (voir par exemple, l'excellent compte-rendu en deux tomes par Brandon Taylor ${ }^{16}$, ou l'exposition remarquablement diverse (y compris en ce qui concerne les contributions à son catalogue), The Great Utopia, au Guggenheim à New York en $1992^{17}$, transforment indubitablement notre conception du passé. Qu'on ait été admirateurs de l'avant-garde bolchévique ou non, on est obligé d'abandonner une vision unilatérale de la culture et de la politique dans le "socialisme réellement existant». Cela pourrait rendre possible la rédemption partielle de la souffrance du passé, en ce que les efforts de la génération révolutionnaire de producteurs culturels en Union soviétique pourraient redevenir significatifs pour notre époque. La multiplicité des débats et des pratiques, non seulement pendant la période héroïque (1917-22) mais aussi pendant les années 1920, et même les années 1930, fournit de nombreuses possibilités pour l'élaboration de nouveaux legs à destination des pratiques actuelles, et indique que, l'appel benjaminien à " la politisation de l'art " possède une " après-histoire » de richesses potentielles que les artistes d'aujourd'hui n'ont même pas commencé à explorer. Il faudrait encourager cette exploration, car beaucoup d'œuvres d'art actuelles soi-disant "politiques» manquent pitoyablement d'inspiration par rapport à l'avant-garde soviétique.

Dans ce contexte, permettez-moi un mot critique sur le travail influent de Boris Groys, émigré russe actuellement professeur à l'université de Munster en Allemagne de l'Ouest. Son livre de 1988, Gesammtkunstwerk Stalin, a produit, il faut le lui concéder, une "constellation" totalement neuve à partir des faits historiques, en affirmant qu'ironiquement, et malgré sa persécution particulière des artistes d'avant-garde, c'était Staline qui avait mis en œuvre leur projet social utopique d'une société totalement transformée, socialiste, et d'un homme nouveau pour l'habiter, poursuivant ainsi la tâche commencée avec enthousiasme et dans un esprit proto-totalitaire par ces mêmes artistes issus de l'avant-garde ${ }^{18}$. Le problème que pose la constellation de Groys est le suivant : elle exemplifie la logique totalitaire qu'elle déplore. En affirmant que toutes les vaches sont grises - que tous les projets culturels socio-utopiques sont fondamentalement totalitaires - elle écarte toute la tradition de l'art politisé, refermant tout débat. Sa position ne fait aucun cas de l'éventail passionnant des nouveaux matériaux que les recherches empiriques continuent de découvrir. Les faits, les étoiles elles-mêmes, ne peuvent aucunement mettre en question le cynisme postmoderne qui alimente sa critique incendiaire. Alors qu'il entend faire exploser de vieux mythes, l'éclairage possible que pourraient offrir certains des nouveaux faits qu'il met à jour est perdue dans la déflagration. Benjamin, lors de son voyage à Moscou, étaient bien particuliers : il ne s'agissait guère 
des good guys du monde sociopolitique comme le prétendent certains travaux universitaires. Au contraire, la ligne dure prise vers la fin des années 1920 contre des tendances culturelles dépolitisées, non ancrées dans la classe ouvrière, n'était pas venue d'en haut, dictée par Staline. Plutôt, l'intolérance ambiante était alimentée par les artistes eux-mêmes, dans des organisations comme la VAPP (Vserossiiskaya Assotsiatsiya Proletariskikh Pisatelei, l'Association panrusse d'écrivains prolétariens). Le contact le plus proche de Benjamin, Bernhard Reich, était membre de cette association au siège de laquelle Benjamin s'est rendu presque quotidiennement lors de son séjour. Fondée en 1920, la VAPP est devenue de plus en plus extrême en 1926-27, militant au nom du prolétariat pour le monopole de la voix culturelle, et réduisant l'opposition au silence. La VAPP était « le protagoniste principal d'une ligne dure dans la littérature » selon l'historienne révisionniste Sheila Fitzpatrick, qui la décrit comme «jeune, effrontée, agressive, consciemment communiste et "prolétaire" dans le sens qu'elle était hostile à la vieille intelligentsia littéraire $»^{19}$ (Benjamin avait 34 ans à l'époque.) Leur position puriste sur la culture révolutionnaire n'était pas partagée par Staline, ce qui n'a pas empêché, comme l'a démontré Fitzpatrick, que celui-ci ait exploité leur énergie de façon opportuniste.

Autrement dit, les communistes desquels Benjamin était le plus proche étaient des radicaux, et non des libéraux : ils croyaient que seules certaines tendances dans les arts étaient progressistes, et ne défendaient pas la liberté d'expression. C'est dans ce contexte que la philosophie de l'Histoire de Benjamin prend tout son sens. Car, pour la plupart, ces artistes de l'avant-garde se sont soumis à la notion progressiste du temps historique au cours des années 1920 (à l'exception intéressante, peut-être, de Malevitch) ${ }^{20}$, c'est-à-dire qu'ils ont accepté la fusion des temporalités artistiques et politiques, fusion contre-nature, car la temporalité de l'avant-garde artistique était, dans le fond, plutôt anarchiste. Lénine n'a toléré cet alignement-là que brièvement (jusqu'en avril 1918). Benjamin, par contraste, n'a jamais accepté la conception avantgardiste du temps. Par conséquent, pour lui, l'intolérance du pluralisme culturel ne pouvait se rabattre sur la rhétorique facile d'un temps "avancé » ou "rétrograde " comme jugements catégoriques. Ceux-ci se devaient d'être argumentés à partir de l'expérience phénoménologique des matériaux eux-mêmes, étant donné l'état du monde - caractérisé, dans la dernière décennie de la vie de Benjamin, par «l'état d'urgence » du fascisme.

L'argument à propos des temporalités différentes est important, et je veux y revenir. Mais laissez-moi d'abord vous donner un autre exemple philologique pour justifier l'importance à long terme des débats en Union soviétique sur l'œuvre de Benjamin ${ }^{21}$. Notamment sur l'essai de 1936, "Le conteur ", qui élabore sa théorie de la fin de l'ère du conte. Pratiquement aucune des analyses universitaires de Benjamin ne se penche sur le conteur particulier dont il est question dans l'essai, à savoir Nikolai Leskov, un écrivain russe du $19^{\mathrm{e}}$ siècle et contemporain de Dostoïevski. Les histoires de Leskov sont ancrées dans la Russie traditionnelle, écrites depuis la perspective de quelqu'un qui a échappé à ce milieu provincial ${ }^{22}$. Et même si les commentateurs de Benjamin se mettaient à lire Leskov, ils ne comprendraient pas pourquoi il traite de $c e$ conteur-ci parmi tous les autres possibles, comme exemple type d'une forme de production culturelle historiquement dépassée. Mais Leskov était, comme le disent les Allemands, "aktuell» dans les débats contemporains ${ }^{23}$. Bien que Benjamin ait avoué n'avoir " aucun désir du tout » de travailler sur l'essai en question, plutôt préoccupé par le livre des Passages, il a accepté d'écrire « Le conteur » à la commande de la revue Orient 
and Okzident en mars 1936, au moment précis où le nom de Leskov était impliqué dans un conflit entre les artistes communistes et la direction soviétique - et ce, après l'adaptation d'une histoire de Leskov par le compositeur Dmitri Chostakovitch, identifié à l'avant-garde artistique révolutionnaire.

L'histoire en question, est elle-même fascinante, comme le titre de l'opéra de Chostakovitch : Lady Macbeth du district du Mzensk. La protagoniste, Katerina Izmailova, est une héroïne typique du $19^{\mathrm{e}}$ siècle notamment à un égard : elle tombe passionnément amoureuse, et sera consommée par cet amour. Mais elle est en même temps bien atypique en ce que plutôt, simplement, que d'en mourir (dénouement de rigueur dans la fiction de l'époque), elle tue au nom de l'amour (comme son homonyme shakespearienne). Elle empoisonne son beau-père qui découvre qu'elle a un amant (le serviteur de son mari). Elle frappe à mort son mari avec un chandelier, et étouffe son beau-neveu (avec l'aide de son amant). Elle tue enfin la nouvelle petite-amie de son amant. Ce n'est qu'alors en se battant avec cette quatrième victime sur la glace de la Volga, qu'elle chute dans le fleuve qui sera leur tombe à toutes les deux. Ce n'était pas le thème sensationnel qui a provoqué la controverse dans les années 1930, mais plutôt l'interprétation moderniste, post-narrative de Chostakovitch.

Quand l'opéra s'est ouvert à Leningrad en 1934, il a été largement acclamé et loué par la presse officielle pour ses innovations musicales et théâtrales. Sergei Eisenstein s'en est servi dans ses cours comme exemple réussi de construction de la mise en scène ${ }^{24}$. Mais en janvier 1936, Staline et Molotov ont assisté à une performance à Moscou par la Seconde Compagnie du Ballet du Bolshoï. Deux jours plus tard, l'opéra a été dénoncé avec véhémence dans la Pravda comme "déformation gauchiste", où "le chaos remplace la musique $»^{25}$. Chostakovitch était abasourdi et secoué. L'incident a connu une grande publicité à l'international, car l'opéra avait déjà été monté en Europe et aux États-Unis ${ }^{26}$. Dans ce contexte, l'impact de l'argument de Benjamin dans "Le conteur » (commandé deux mois après la dénonciation de la Pravda) aura été de défendre un artiste communiste contemporain contre les critiques politiques antimodernistes des dirigeants soviétiques. On est bien loin de la simple lamentation devant la mort d'une forme littéraire prémoderne, interprétation habituelle de cet essai par les spécialistes de Benjamin...

19 Terminer la discussion ici serait toutefois recourir à l'historicisme pour critiquer les interprétations contemporaines; j'ai déjà affirmé que cette alternative n'était pas valable. Nous n'avons par ailleurs aucune preuve que c'était l'intention de Benjamin que d'intervenir par son essai dans la controverse autour de Chostakovitch et nous n'en avons de toute manière pas besoin pour nous intéresser à une vérité qui, comme l'a dit Benjamin, n'est justement pas intentionnelle. Dans l'introduction du Trauerspiel, il écrit : «La vérité, c'est la mort de l'intention » [Die Wahrheit ist der Tod der Intention]. Ce qui compte davantage que l'intention ou non de Benjamin par rapport aux controverses soviétiques, c'est qu'il est éclairant pour nous de la supposer. En suggérant une telle constellation, je voudrais revenir, comme promis, à la question de la temporalité et de la philosophie de l'Histoire.

Le livre récent de Peter Osborne, The Politics of Time, m'a poussé à réfléchir philosophiquement sur la dimension politique de divers concepts de temporalité, surtout la partie qui critique explicitement ma lecture de Benjamin ${ }^{27}$. Je pense qu'il a raison de décrire le concept de temps révolutionnaire chez Benjamin comme une rupture « expérientiellement vécue » (phenomenally lived), comme une interruption de 
la vie de tous les jours, par-là fondamentalement différente de la temporalité cosmologique qui marque la conception hégélo-marxiste, et qui était aussi celle de Lénine et du Parti communiste. Mais il est problématique d'assimiler, comme le fait Osborne, la conception benjaminienne du temps à la temporalité de l'avant-garde problématique parce que cette distinction théorique fait abstraction de l'histoire réelle, et en tant que marxiste, voire en tant que philosophe marxiste, Osborne n'aurait pas dû faire une telle abstraction. Osborne écrit que l'expérience benjaminienne du "maintenant» (qu'il appelle l'«être-maintenant», dans un geste heideggérien douteux) est « une forme d'expérience avant-gardiste. Car l'avant-garde n'est pas ce qui est le plus avancée historiquement au sens où elle (...) a le plus d'Histoire derrière elle ${ }^{28}$

21 Pourtant, voilà précisément comment l'avant-garde se comprenait. Rappelons-le brièvement: le terme "avant-garde " a émergé en France au milieu du $19^{\mathrm{e}}$ siècle $^{29}$. À l'époque, il s'appliquait à la fois au radicalisme politique et au radicalisme culturel, car les deux souscrivaient, dans un esprit saint-simonien, à l'idée de l'Histoire comme progrès. Vers la fin du siècle, dans le climat du modernisme artistique, centré sur le Paris bourgeois (où vivaient beaucoup d'artistes russes avant la Révolution), «l'avantgarde » a pris un sens plus spécifiquement culturel. Bien que la plupart (mais pas tous !) de ses membres soient plus ou moins à gauche, le terme n'impliquait pas une prise de position politique. Il désignait plutôt les artistes aliénés de la culture bourgeoise établie et à la pointe de l'histoire culturelle : la question de joindre cette sensibilité au soutien d'un parti politique ne se posait pas.

Elle a fini par se poser, cependant. Du moins pour l'avant-garde russe, à la suite du succès bolchévique en octobre 1917. Lénine a toute de suite parlé de cet évènement révolutionnaire dans les termes d'une temporalité cosmologique. Octobre 1917 était pour lui un évènement historique mondial, le point culminant d'un continuum dans lequel le Paris bourgeois avait joué un rôle primordial, mais seulement dans le passé. On a considéré la Révolution française et la Commune de Paris comme autant d'étapes sur le chemin. Cette vision de l'Histoire devait être fixée par l'art. Lénine a lancé le Plan d'une propagande monumentale, dressant la liste approuvée des « combattants pour le socialisme", des figures historiques de l'Europe occidentale et de la Russie, qui méritaient d'être commémorées par des monuments publics dans l'espace urbain. Les bolchéviques ont mis un point d'honneur à tenter d'intégrer l'avant-garde dans leurs programmes culturels. Mais du côté des artistes, tandis qu'ils soutenaient généralement la révolution d'Octobre, intellectuellement néanmoins, leur position demeurait parfois ambiguë. Beaucoup d'artistes importants de l'avant-garde avaient adopté des positions explicitement "anarchistes " (cela était particulièrement vrai dans le printemps 1918 quand, sous la pression de la guerre renouvelée contre l'Allemagne, la direction léniniste a commencé à réprimer les anarchistes) ${ }^{30}$, créant un malaise parmi les artistes les plus «radicaux» quant au coût en termes de liberté créative de leur collaboration étroite avec toute organisation d'État, y compris les organisations nouvellement créées. Ici, la politique des temporalités conflictuelles devient importante.

23 C'était justement le parti-pris de l'Histoire-comme-progrès qui a mené les producteurs culturels radicaux à supposer que la révolution politique et la révolution artistique étaient les deux faces d'une même médaille. La prétention de l'avant-garde à être le destinataire historique de l'art trouvait sa légitimité dans la soumission à la 
temporalité cosmologique du Parti, mais ce faisant, sa propre «vérité » s'est historicisée. Déjà au milieu des années 1920, en Union soviétique, on jugeait l'avantgarde passéiste. Tout art qui n'allait pas dans le sens du Parti était considéré comme « rétrograde », bourgeois, non prolétarien et, en fin de compte, contre-révolutionnaire. Dès lors que les artistes ont accepté le temps chronologique de l'avant-garde politique, il s'ensuivait qu'être révolutionnaire dans un sens culturel signifiait aussi glorifier les succès du Parti et fermer les yeux sur ses échecs.

On pourrait affirmer que, en dépit de l'appel constructiviste à l'intégration de l'art dans la vie sociale, l'avant-garde a été détruite précisément en essayant de s'accrocher à « l'art » de manière trop tenace, c'est-à-dire de s'accrocher à un continuum historique de l'art qui évoluait en parallèle - avant d'y être asservi - du continuum cosmologique $\mathrm{du}$ progrès historique. Après la révolution d'Octobre, le simple geste de refus qui caractérisait l'avant-garde bourgeoise ne suffisait plus. Les artistes ont pris la décision fatale de regarder en avant plutôt qu'en arrière et d'avancer triomphalement vers l'avenir, aux côtés du pouvoir politique. Le seul sujet à débat concernait la vitesse relative de cet accompagnement: il s'agissait de savoir si, comme l'affirmaient les constructivistes Tatline et Lissitzky, la pratique artistique était en avance sur le Parti, ou si, comme l'a écrit Trotski, l'art se trouvait toujours « dans le fourgon à bagages » de l'Histoire. En acceptant la conception cosmologique du temps révolutionnaire de l'avant-garde politique, l'avant-garde artistique a renoncé à la temporalité qu'Osborne veut lui attribuer, la temporalité benjaminienne d'interruption, d'éloignement, d'arrêt. C'est-à-dire qu'elle a abandonné l'expérience phénoménologique de la pratique avantgardiste. Il faut comprendre cette dernière non seulement comme une stratégie pour subvertir l'ordre bourgeois, mais aussi comme une pratique culturelle fondamentale à toute société future digne du qualificatif de " socialiste ».

Le temps révolutionnaire doit donc être appréhendé comme une expérience temporelle, éternellement en opposition au continuum chronologique de l'Histoire, et non moins en opposition au geste répétitif de la mode, du « nouveau » qui se fait passer pour l'avant-garde aujourd'hui. La culture socialiste et la culture d'avant-garde doivent être repensées dans les termes de cette temporalité, comme la construction constante de constellations qui font arrêter le temps; comme la lutte constante contre les dirigeants économiques et politiques qui, incultes, prédisent mal le futur en extrapolant le présent; comme l'opposition constante aux faiseurs de mode pour qui le temps est, comme une marchandise, doté d'une obsolescence programmée.

Le seul pouvoir qu'il nous reste alors que nous autres, voyageurs à bord du train de l'Histoire, cherchons le frein de secours, est celui qui nous vient du passé - un passé qui, sans nos efforts, sera oublié. Un aspect du passé que nous risquons le plus d'oublier est la simplicité apparente avec laquelle le processus de capitulation culturelle a eu lieu. Il s'est banalement présenté sous les traits d'une nécessaire adaptation aux nouvelles tendances intellectuelles: accepter le marché, rester au fait du monde tel qu'il va, suivre tant que faire se peut la mode. Dans notre époque, cela implique en substance de rejeter l'autre histoire du $20^{e}$ siècle, celle du socialisme, qui aurait " échoué ". Mais ce serait alors consentir à la dernière version du mythe de progrès, la présomption erronée selon laquelle ceux qui, à l'Est, ont été " défaits » par l'Histoire, n'ont rien à apprendre aux nouveaux barbares triomphants de l'Occident.

Mais alors, qu'est-ce que nous fichons ici? L'épreuve de vérité pour la production intellectuelle réside dans son influence sur le monde extérieur, et non dans ce qui se 
passe dans une enclave académique comme celle dans laquelle nous nous trouvons actuellement. Benjamin a lui- même établi un critère pour ses travaux : qu'ils soient «totalement inutilisables pour les buts du fascisme $»^{31}$. Qui d'entre nous pourrait dire que son travail est totalement inutilisable pour les buts du nouvel ordre mondial, où l'exploitation de classe est flagrante, mais où le langage pour la décrire est en ruines ? Bien entendu, nous serions horrifiés si les décisions concernant l'embauche et de la promotion à l'université étaient prises sur la base de la contribution de nos travaux à la lutte des classes. Voici cependant la vérité qui dérange : ce genre de décision s'appuie d'ores et déjà sur le principe que nos travaux n'ont rien à voir avec la lutte des classes. Et cela, mes amis, est problématique.

\section{NOTES}

1. Cf. Passagen-Werk : "L'histoire que montraient des choses "comme elles étaient réellement" était le narcotique $\mathrm{du}\left[19^{\mathrm{e}}\right]$ siècle ».

2. Argument avancé avec vigueur par Irving Wohlfarth, "Smashing the Kaleidoscope", in Michael P. Steinberg, dir., Walter Benjamin and the Demands of History, Ithaca (Cornell University Press) 1996, p. 198, 204-05.

3. La connaissance intime qu'avait Benjamin des débats en Union soviétique a commencé lors de sa relation avec Asja Lacis, dont la passion intellectuelle avait une profonde influence sur lui. Leurs discussions politiques étaient vives et interminables. La pratique de Lacis en tant que directrice de théâtre lui a donné l'exemple d'une alternative communiste au théâtre bourgeois. Benjamin a continué à discuter de ces questions avec Bertolt Brecht, rencontré en 1929 par l'intermédiaire de Lacis. Tout aussi significatif est le fait que son frère Georg (duquel il était très proche) a rejoint le Parti communiste allemand dans les années 1920. Georg Benjamin fut arrêté et incarcéré en 1933, puis libéré. Ensuite, il a écrit pour la presse underground, traduisant des articles anglais, français et russes, couvrant le Front populaire et le septième congrès mondial de l'Internationale communiste en juillet 1935. Incarcéré de nouveau, il fut transféré au camp de Mauthausen, où il est mort en 1942. On l'a décrit comme « l'alter ego politique de Walter » (Cf. Momme Broderson, Walter Benjamin. A Biography, London : Verso, 1996, 208-09).

4. Gershom Scholem, préface à Walter Benjamin, Moscow Diary, Cambridge, Mass. (Harvard University Press), 1986, p. 6.

5. L'éditeur de Benjamin, Rolf Tiedemann, remarque qu'il n'a pas trouvé la moindre preuve du fait que cette conférence a eu effectivement lieu, mais dans sa correspondance, Benjamin dit qu'il a écrit le texte à cette fin.

6. Voir mon ouvrage, The Dialectics of Seeing: Walter Benjamin and the Arcades Project, Cambridge, Mass. (MIT Press), 1989, p. 124-5.

7. Ivan Puni (1919), cité in Christina Lodder, Russian Constructivism, New Haven (Yale University Press), 1983, p. 48.

8. Pour sa réaction à la production controversée par Meyerhold de la pièce de Gogol, L'Inspecteur général, voir Moscow Diary, p. 32-34. Pour sa réaction au film d'Eisenstein, Potemkin, voir II, p. 751-55. 
9. Walter Benjamin, Understanding Brecht, London (NLB), 1966, p. 102. L'image de l'écrivain comme ingénieur introduit le texte de 1926, One Way Street and other Writings, London (Verso, 2021 ; NLB, 1979).

10. Le centenaire des "phalanstères" de Fourier a été commémoré à Paris en 1932. Pour son importance dans la Russie postrévolutionnaire, voir S. Frederick Starr, Melnikov: Solo Architect in a Mass Society, Princeton (Princeton University Press), 1978, p. 50-51.

11. Voir note 8.

12. «Percevoir l'aura d'un objet que nous regardons signifie l'investir avec la capacité de nous regarder en retour " (je traduis), (Illuminations: Essays and Reflections, New York (Schoken, 1969), p. 188). Pour Brecht sur Benjamin, voir mon livre, Dialectics of Seeing, op. cit., p. 246 ; pour la théorie de l'objet socialiste, voir le travail fondateur de Christina Kaier, cité ci-après, note 14.

13. La salle de lecture ouvrière de Rodchenko ainsi que la maquette de Tatlin furent exposées pour le Monument à la 3e Internationale, dans le pavillon soviétique dessiné par l'architecte Melnikov.

14. Cité in Christina Kaier, « Rodchenko in Paris », October, 75, (hiver 1996), p. 30.

15. Franco Borsi, The Monumental Era: European Architecture and Design 1929-1939, New York (Rizzoli), 1986.

16. Brandon Taylor, Art and Literature under the Bolsheviks, 2 tomes. London (Pluto Press), 1992.

17. The Great Utopia. The Russian and Soviet Avant Garde, 1915-1932, New York (Guggenheim Museum), 1992.

18. Boris Groys, Gesammtkunstwerk Stalin: Die gespaltene Kultur in der Sowjetunion, Munich (Carl Hanser Verlag), 1988.

19. Sheila Fitzpatrick, The Cultural Front: Power and Culture in Revolutionary Russia, Ithaca, NY (Cornell University Press), 1992, p. 104.

20. Malevich a délibérément commencé à brouiller la chronologie de ses tableaux vers la fin des années 1920, faisant penser à un " développement » en temps virtuel uniquement. Même avec l'altération des faits, son style a adopté une temporalité cyclique : ses tableaux tardifs se sont retournés au style et au contenu des thèmes paysans d'avant-guerre; ses dernières œuvres, incluant un autoportrait, mettent des figures réalistes en costume de la Renaissance.

21. Je dois cet exemple à Jennifer Tiffany, du département de planification régionale à Cornell University.

22. Voir Hugh McLean, Nikolai Leskov: The Man and His Art, Cambridge, Mass. (Harvard University Press), 1977.

23. Benjamin avait d'abord été exposé à Leskov en 1928 à travers une nouvelle édition allemande de son œuvre. Mais il semble bien que ce soit la revue Orient and Okzident qui ait été à l'initiative de l'article sur Leskov en mars 1936.

24. Voir David Bordwell, The Cinema of Eisenstein, Cambridge, Mass (Harvard University Press), 1993, p. 156-57.

25. Fitzpatrick, op. cit, p. 187. Une traduction française de l'article de la Pravda se trouve dans Krzysztof Meyer, Dmitri Chostakovitch, Fayard, 1994, p. 203-04 (NdT).

26. Bordwell, op. cit., p. 156.

27. Peter Osborne, The Politics of Time: Modernity and the Avant-Garde, London (Verso) 1995, p. 150-53.

28. Ibid., p. 150.

29. Voir Linda Nochlin, «The Invention of the Avant-Garde: France, 1830-80 », in Thomas B. Hess et John Ashbery (dirs.), Avant-Garde Art, London (Collier-Macmillan), 1968, p. 5.

30. Hubertus Gassner, "The Constructivists: Modernism on the Way to Modernization », in The Great Utopia, p. 302-305.

31. Benjamin, préface à "The Work of Art in the Age of Mechanical Reproduction " in Illuminations, op. cit., p. 216. 


\section{AUTEURS}

\section{SUSAN BUCK-MORSS}

Professeure émérite de philosophie politique à la City University of New York (CUNY). 